\title{
The binary properties of the pulsating subdwarf B eclipsing binary PG 1336-018 (NY Virginis) ${ }^{\star}$
}

\author{
M. Vučković ${ }^{1}$, C. Aerts ${ }^{1,2}$, R. Østensen ${ }^{1}$, G. Nelemans ${ }^{2}$, H. Hu ${ }^{1,2}$, C. S. Jeffery ${ }^{3}$, V. S. Dhillon ${ }^{4}$, and T. R. Marsh ${ }^{5}$ \\ 1 Institute for Astronomy, K.U. Leuven, Celestijnenlaan 200D, 3001 Leuven, Belgium \\ e-mail: maja.vuckovic@ster.kuleuven.be \\ 2 Department of Astrophysics, Institute of Mathematics, Astrophysics and Particle Physics (IMAPP), Radboud University, \\ 6500 GL Nijmegen, The Netherlands \\ 3 Armagh Observatory, College Hill, Armagh, BT61 9DG Northern Ireland \\ 4 Department of Physics and Astronomy, University of Sheffield, Sheffield S3 7RH, UK \\ 5 Department of Physics, University of Warwick, Coventry CV4 7AL, UK
}

Received 26 January 2007 / Accepted 24 May 2007

\section{ABSTRACT}

\begin{abstract}
Aims. We present an unbiased orbit solution and mass determination of the components of the eclipsing binary PG $1336-018$ as a critical test for the formation scenarios of subdwarf B stars.

Methods. We obtained high-resolution time series VLT/UVES spectra and high-speed multicolour VLT/ULTRACAM photometric observations of PG 1336-018, a rapidly pulsating subdwarf B star in a short period eclipsing binary.

Results. Combining the radial velocity curve obtained from the VLT/UVES spectra with the VLT/ULTRACAM multicolour lightcurves, we determined numerical orbital solutions for this eclipsing binary. Due to the large number of free parameters and their strong correlations, no unique solution could be found, only families of solutions. We present three solutions of equal statistical significance, two of which are compatible with the primary having gone through a core He-flash and a common-envelope phase described by the $\alpha$-formalism. These two models have an sdB primary of $0.466 M_{\odot}$ and $0.389 M_{\odot}$, respectively. Finally, we report the detection of the Rossiter-McLaughlin effect for PG 1336-018.
\end{abstract}

Key words. stars: subdwarfs - stars: binaries: eclipsing - stars: general - stars: variables: general - stars: oscillations stars: individual: PG 1336-018

\section{Introduction}

The subdwarf B (sdB) stars are generally acknowledged to be core helium burning stars with a canonical mass of approximately $0.5 M_{\odot}$. Their thin, inert hydrogen envelope $\left(M_{\text {env }} \lessgtr 0.02 M_{\odot}\right)$ places them on the hot extension of the Horizontal Branch (HB), the so-called Extreme Horizontal Branch (EHB). Since the hydrogen envelope is too thin to sustain nuclear burning, these stars will not go through the Asymptotic Giant Branch and Planetary Nebula phases. Instead, when their core helium has run out, they will enter a He-shell burning phase, where they expand and heat up, making them appear as sdO stars before they evolve directly onto the white dwarf cooling sequence. Even though the models describing the future evolution of the sdB stars are generally accepted (e.g. those of Dorman et al. 1993), the current evolutionary state of the sdB stars is still poorly understood. The fact that $\mathrm{sdB}$ stars must have lost almost all of their hydrogen layer at exactly the same time when the helium core has attained the minimum mass required for the helium flash to occur, makes them enigmatic from an evolutionary point of view. To loose such an amount of mass, they must suffer considerable mass loss during the red giant branch (RGB) phase, and most probably also during the helium core flash. The most fundamental missing piece to our understanding of the evolution

* Based on observations collected at the European Southern Observatory, Chile. Program ID: 075.D-0174. of the sdB stars, apart from the physics during the helium core flash, is the nature and physics behind this mass loss (Fusi-Pecci \& Renzini 1976).

In recent years it has been discovered that a significant fraction of sdBs are in binaries. Maxted et al. (2001) found that about two-thirds of the sdB stars in the field are members of binaries. Napiwotzki et al. (2004) found a binary fraction of $40 \%$ among stars in the SPY (Supernova type Ia Progenitor) survey sample, while Morales-Rueda et al. (2006) found 48\% in a sample from the Edinburgh-Cape (EC) survey. Many of the binary sdBs are found to be in short period systems with periods from a few hours to several days, with companions being either white dwarfs or M-dwarfs (Morales-Rueda et al. 2003). The peculiar frequency of binarity has been an important constraint on evolutionary population synthesis theory, and has led to the acknowledgment that the binarity has to play a key role in the formation channels for sdB stars. There are several binary mechanisms proposed by (Han et al. 2002, 2003, and references therein) as formation channels for sdB stars:

1. common envelope ejection, leading to short-period binaries with periods between 0.1 and 10 days and an $\mathrm{sdB}$ star with a very thin hydrogen envelope, and with a mass distribution that peaks sharply at $0.46 M_{\odot}$. Depending on the secondary, a main-sequence star or a white dwarf, the subchannels are called the first CE ejection channel and the second CE ejection channel, respectively; 
2. stable Roche lobe overflow, resulting in similar masses as in 1. but with a rather thick hydrogen-rich envelope and longer orbital periods between 10 and 100 days;

3. double helium white dwarf mergers giving rise to single $\mathrm{sdB}$ stars with a wider distribution of masses.

Detailed investigation of sdB binaries is crucial in order to determine their masses for comparison with the theoretically proposed evolutionary channels. New momentum in the efforts to resolve the evolutionary paths of sdB stars came a decade ago, after the discovery that some of them pulsate (Kilkenny et al. 1997). This has opened up a new window into their interiors via the techniques of asteroseismology and stimulated a burst of research. Extensive search campaigns have revealed two classes of pulsating $\mathrm{sdB}$ stars known as short period sdB variables ( $\mathrm{sdBV}$ or V361 Hya stars, formerly EC 14026 stars, after the prototype) and long period sdB variables known as PG 1716 stars (or lpsdBV stars, Green et al. 2003).

The sdBV stars, discovered by Kilkenny et al. (1997) and independently theoretically predicted by Charpinet et al. (1996), are low amplitude multimode pulsators with typical periods ranging between 100-250 s. Their pulsation amplitudes are generally of the order of a few hundredths of a magnitude. The short periods, being of the order of and shorter than the radial fundamental mode for these stars, suggest that the observed modes are low-order, low-degree $p$-modes (Charpinet et al. 2000). The 39 known sdBV stars occupy a region in the $T_{\text {eff }}-\log g$ plane with effective temperatures between $28000 \mathrm{~K}$ and $36000 \mathrm{~K}$ and surface gravities $(\log g)$ between 5.2 and 6.2.

The detailed asteroseismological modelling of sdBV stars is hampered by the fact that there are too few pulsational frequencies to fit those predicted from non-rotating or rigidly rotating models (Brassard et al. 2001; Charpinet et al. 2005; Randall et al. 2005). However, the observed frequency spectra are too dense to be accounted for by only low-degree $(\ell \leq 2)$ modes. In order to have a unique asteroseismological model we need to have accurate pulsation frequencies and an unambiguous identification of the modes of oscillation (spherical wavenumbers $\ell$ and $m$ ). Thanks to multisite campaigns by the $\mathrm{WET}^{1}$ devoted to resolving the frequency spectrum of sdBV stars in the last decade, we do have extensive and reliable frequency lists for several sdBVs. The problem lies in the second requirement mentioned above, the unambiguous mode identification. There are only two ways this can be achieved: through line profile variations (Aerts \& Eyer 2000) or the amplitude ratio method (Dupret et al. 2003; Randall et al. 2005).

As sdBV stars are quite faint (the brightest one is $m_{\mathrm{B}}=11.8$ ) and their periods are very short, the line profile variation method poses a real challenge considering the low $\mathrm{S} / \mathrm{N}$ that accompanies any high-resolution time-resolved spectroscopy, even with the biggest telescopes available. Hence, the line profile variation method has not yet been reliably applied to any sdBV star. The amplitude ratio method is not problem free either. Due to the very low pulsational amplitudes, the photometric errors are usually too large for unambiguous identification of the spherical degree $\ell$ of the modes, especially to distinguish between $\ell=0$, 1 and 2 modes (Jeffery et al. 2005).

Among the binary sdB stars, four eclipsing sdB systems have been discovered that all show a deep and strong reflection effect, with very short orbital periods in the rather narrow range of 130-170 min. Such short orbital periods imply that they must have evolved through binary mass transfer and common envelope evolution. Out of these four systems, namely HW Vir

\footnotetext{
${ }^{1}$ http://wet.physics.iastate.edu/
}

(Wood et al. 1993; Menzies \& Marang 1986), NY Vir (Kilkenny et al. 1998) (hereafter PG 1336-018), HS 0705+6700 (Drechsel et al. 2001) and HS 2231+2441 (Østensen et al. 2007), only one system contains a rapidly pulsating $\mathrm{sdB}$ star as a primary: PG 1336-018. As such, this system provides a natural laboratory for detailed evolutionary and asteroseismic analyses, which is the purpose of our project.

PG 1336-018 was classified as an sdB star in the PalomarGreen survey (Green et al. 1986) and shown to be a close eclipsing binary with short-period multimode light variations by Kilkenny et al. (1998). Assuming the primary mass to be the canonical sdB mass of $0.5 M_{\odot}$, Kilkenny et al. (1998) find that the secondary must be a mid-M dwarf with a mass of about $0.15 M_{\odot}$. Soon after its discovery, PG 1336-018 was a target of two Whole Earth Telescope (WET) campaigns, Xcov 17 in April 1999 (Kilkenny et al. 2003) and Xcov 21 in April 2001. These white light data resolved more than 20 frequencies in the temporal spectrum (Kilkenny et al. 2003) in the range from 5000 to $8000 \mu \mathrm{Hz}$. Even though the frequency content of the star is thus known very precisely, an adequate asteroseismic model is still lacking mainly due to the lack of an unambiguous mode identification. The colour behaviour is needed for photometric mode identification to identify the spherical degree $\ell$ of the modes and to discriminate between the numerous possible seismic models. To further reduce the allowable seismic model space we need to examine line profile variations due to the pulsations in order to disentangle the azimuthal wavenumber $m$. Only with the accurate pulsation frequencies and an unambiguous mode identification can the asteroseismology provide the accurate mass estimate needed for confrontation with those predicted from the formation scenarios for sdB stars.

PG 1336-018, being the only rapidly pulsating sdB star in an eclipsing binary, is the only star with enough potential to confront the proposed evolutionary scenarios, as the eclipses help constrain the inclination and radii. Therefore we study PG 1336-018, this time armed with new multicolour photometric and spectroscopic VLT data. In this paper we present the new data and the orbital solution. This is the first step toward our ultimate goal, an accurate mass determination of PG 1336-018 and a critical test of current stellar evolution theory.

\section{Observations and data reduction}

\subsection{Photometry}

PG 1336-018 $\left(\alpha_{2000}=13: 38: 48.2, \quad \delta_{2000}=-02: 01: 49.0, \quad m_{\mathrm{V}}=\right.$ 13.4) was observed on the night of May 18/19 2005 using the ULTRACAM camera attached to the ESO VLT UT3 (Melipal) at Paranal Observatory, Chile. ULTRACAM is a high-speed threechannel CCD camera specifically designed for fast photometry programmes (Dhillon \& Marsh 2001). We gathered two full orbital cycles, about $5 \mathrm{~h}$, of PG 1336-018 simultaneously in three filters $r^{\prime}, g^{\prime}$ and $u^{\prime}$ of the SDSS system (Fukugita et al. 1996). The seeing (around 0.9 arcsec) was variable during the night and getting worse toward the end of the run. The exposure time was $0.5 \mathrm{~s}$ in the beginning of the run, but due to poorer seeing was increased to $1 \mathrm{~s}$ to improve the $\mathrm{S} / \mathrm{N}$. This did not deteriorate our temporal resolution significantly, since the shortest period found in PG 1336-018 is $97 \mathrm{~s}$ (Kilkenny et al. 2003). To achieve $1 \mathrm{~s}$ time resolution, it was necessary to define 2 windows on each of the 3 ULTRACAM chips. One window was placed around PG1338-018, and another on a nearby comparison star. The dead-time of the observation was $24 \mathrm{~ms}$. 


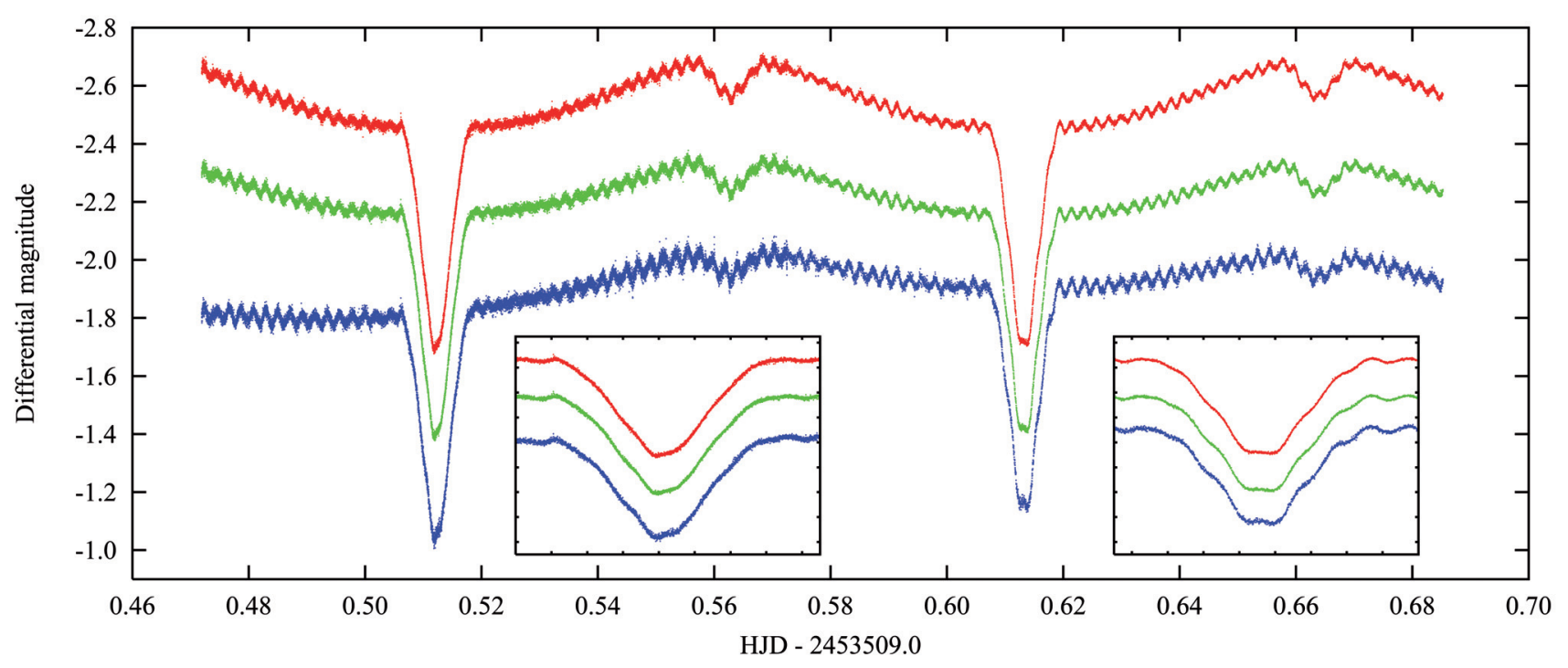

Fig. 1. ULTRACAM/VLT $r^{\prime}$ (upper), $g^{\prime}$ (middle) and $u^{\prime}$ (bottom) lightcurves of the eclipsing sdBV star PG $1336-018$ from 2005 May 18/19. The insets show enlarged sections of the two primary eclipses, where pulsations are clearly visible. The differences between the two consecutive primary eclipses, apart from the noise, are due to the beating of the modes and different phases covered during the eclipse. The shape of the $u^{\prime}$ lightcurve is discussed in the text. The ordinate is the differential magnitude, and the abscissa is Fractional Julian Date.

All data frames were reduced using the ULTRACAM pipeline reduction software (Dhillon \& Marsh 2001). Care was taken to select the most optimal choices offered in the reduction software. The "normal" extraction method with the "variable" aperture sizes, as they track local changes in the seeing disk, gave the best results. Several apertures were tried out and an aperture of 1.7 times the FWHM gave the highest $\mathrm{S} / \mathrm{N}$ for $r^{\prime}$ and $g^{\prime}$ band. The star counts were divided by the comparison star counts and converted to obtain a differential magnitude $(V-C)$ in each filter. As both the target and the comparison star were in the same field, differential photometry accounted well for the variations in the sky transparency and extinction in $r^{\prime}$ and $g^{\prime}$ band. Unfortunately, the only comparison star within ULTRACAM's 2.6 arcmin field of view on the VLT is very faint in the blue, resulting in poorer differential photometry in the $u^{\prime}$ compared to the $r^{\prime}$ and $g^{\prime}$ band. Therefore, a wider aperture had to be used for the $u^{\prime}$ band. Due to the faintness of the comparison star in $u^{\prime}$, its $g^{\prime}$ band lightcurve was used to make the differential $u^{\prime}$ lightcurve. This gave a satisfactory result in the sense that both the pulsations and the eclipses were recovered, but it introduced an unreliable slope in the first part of the $u^{\prime}$ lightcurve (see Fig. 1). Therefore, we did not rely on the $u^{\prime}$ lightcurve for the orbital analysis. However, we did use the second part of the $u^{\prime}$ lightcurve to cross-check our results, as well as for the frequency analysis (see Sect. 4.2).

The times in the data frames were converted to JD and barycentrically corrected. Differential $(V-C)$ lightcurves for $r^{\prime}$, $g^{\prime}$ and $u^{\prime}$ were constructed from a set of more than 80000 science frames. The $r^{\prime}, g^{\prime}$ and $u^{\prime}$ lightcurves are plotted in Fig. 1, where we can see a clear sign of the pulsations of the primary component in all the phases of the binary orbit, even during the primary eclipse. A strong reflection-like effect $\left(0.2\right.$ mag in $g^{\prime}$ and $0.25 \mathrm{mag}$ in $r^{\prime}$ ) is evident. This effect, characteristic of all binary systems containing an sdB star and a cool M-dwarf companion in rotationally locked orbit, is due to the high contrast in the temperatures between the heated and unheated hemispheres of the M-dwarf.

\subsection{Spectroscopy}

Even though PG 1336-018 was a target of several photometric campaigns, its faintness relative to the rapid oscillations has prevented any reasonably good time-resolved spectroscopy. The short pulsation periods require very short integration times. There were two attempt so far with the aim of detecting the pulsational radial velocities (Woolf et al. 2003) and identification of the pulsation modes from the wavelength dependency of the amplitudes (Dreizler et al. 2000), both with a null result.

A time-series of 399 high resolution spectra were taken over a period of $\sim 9 \mathrm{~h}$, covering about 3.7 full orbits, on the night of April 28, 2005 using the Ultraviolet Visual Echelle Spectrograph (UVES) on the VLT UT2 (Kueyen) at the Paranal Observatory, Chile. Only the blue arm was used, with wavelength coverage from 3900 to $5000 \AA$, and the slit width of 1 arcsec at a resolution of 46890 . Each spectrum was integrated for $45 \mathrm{~s}$ which, with the ultra fast read-out of about $23 \mathrm{~s}$ we used, gave a time resolution of $68 \mathrm{~s}$. Dome flat-fields and bias calibration frames were taken at the beginning and at the end of the night, and ThAr exposures were taken before and after the run.

Due to the very low signal we got for such a short exposure and the ultra fast read-out mode used, the UVES reduction pipeline did not give satisfactory results. Therefore, we developed a non-standard reduction method, using the ESO-MIDAS package. This provided a factor of $\sim 2$ increase in the $S / N$ ratio of the reduced spectra, compared to those produced by the pipeline. The bias calibration frames had an offset between the upper and the lower part, due to the ultra fast read-out mode used. After careful examination of each bias frame, we proceeded as follows. First we examined the interorder space of each science frame (by taking the median of the box) to determine these offsets which were then subtracted from the science frames. Then the science frame was corrected for cosmic rays, extracted and background corrected (which was smoothed to reduce the noise). Since, in our case, the sky background contributes most to the noise, we used optimal extraction which gave better $\mathrm{S} / \mathrm{N}$, as suggested by Mukai (1990). Then the science frames were flat-field 


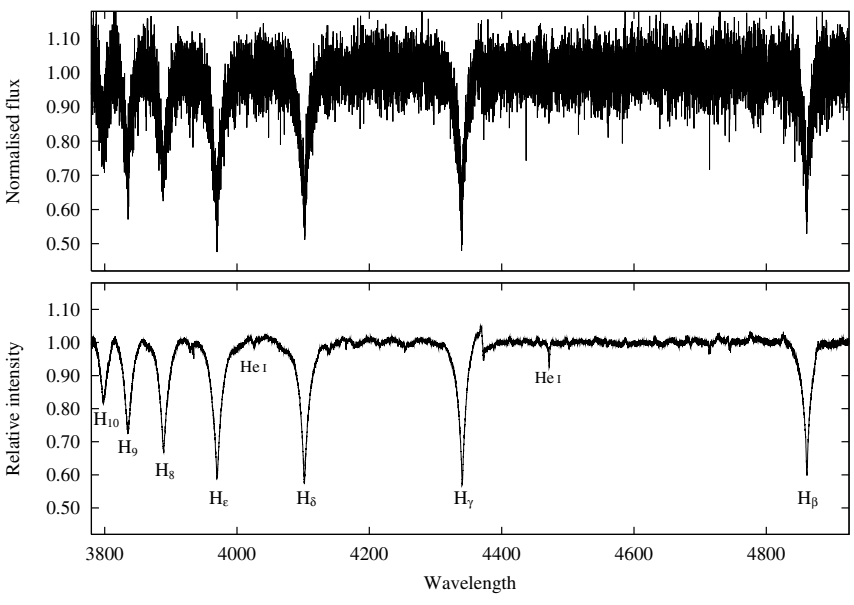

Fig. 2. A typical single UVES/VLT spectrum of PG 1336-018 from our VLT run on 2005 April 28 (top) and the coadded spectrum (bottom), produced by combining all the 399 available spectra after shifting according to the orbital radial velocity solution. The Balmer lines are indicated together with the helium lines used for the determination of physical parameters. Discontinuities due to imperfect merging of spectral orders only become evident in the high-S/N combined spectrum.

corrected, wavelength calibrated and, finally, the orders were merged. Since the spectra were oversampled we have rebinned them in an optimal way such that the $\mathrm{S} / \mathrm{N}$ increased without compromising the resolution. Finally, the science frames were normalized.

A typical individual spectrum of PG 1336-018 is shown in the top panel of Fig. 2. The bottom panel of Fig. 2 shows the coadded orbit-corrected spectrum (see Sect. 4.1). Despite our extensive effort to achieve the optimal reduction scheme, the extraction and merging of the orders is not perfect. This is due to the fact that the Echelle order discontinuities do not behave "consistently" under a low signal. This leads to some jumps and wiggles seen in the continuum of the coadded spectrum and particularly in the red wing of $\mathrm{H}_{\gamma}$. For this reason we did not make use of this line in the merged spectrum for the spectroscopic parameter determination discussed below.

In the blue wavelength range covered by our data no sign of any spectral feature from the cool companion can be seen, confirming the results of Woolf et al. (2003). Due to the large difference in effective temperatures (about a factor of 10, see Sect. 4) the hot sdBV star dominates the spectrum even in the primary eclipse.

\section{RV determination}

Our spectra allow us to produce a radial velocity (RV) curve, with an excellent phase coverage, from which we can independently determine the orbital period $(P)$ and semi-amplitude $\left(K_{1}\right)$ of this eclipsing binary. As we are dealing with a low $\mathrm{S} / \mathrm{N}$, we determined RVs from the spectra trying out several different methods. The best results were obtained by using molly- a software package, which fits two Gaussian profiles to the Balmer line profiles $^{2}$. This allows good treatment of both the broad wings and the sharper core at the same time. This gave better results than any of the other methods we have tried.

We have measured the RVs of the highest $\mathrm{S} / \mathrm{N}$ lines in the spectrum, namely $\mathrm{H}_{\epsilon}, \mathrm{H}_{\delta}, \mathrm{H}_{\gamma}$ and $\mathrm{H}_{\beta}$, using this package.

${ }^{2}$ http://deneb.astro. warwick.ac.uk/phsaap/software/ molly/html/INDEX.html

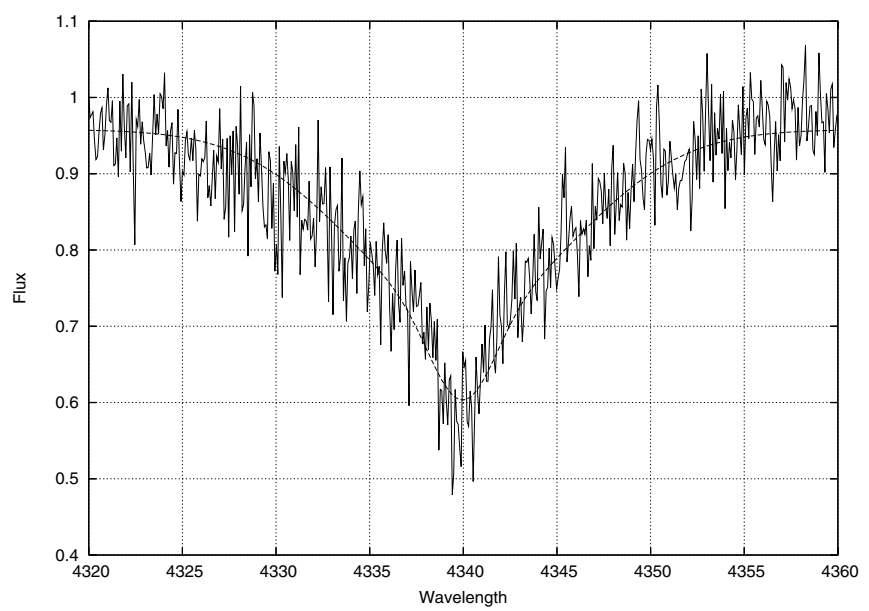

Fig. 3. A sample fitting of two Gaussians to the observed $\mathrm{H}_{\gamma}$ line (the same spectrum as the one shown in Fig. 2) using molly.

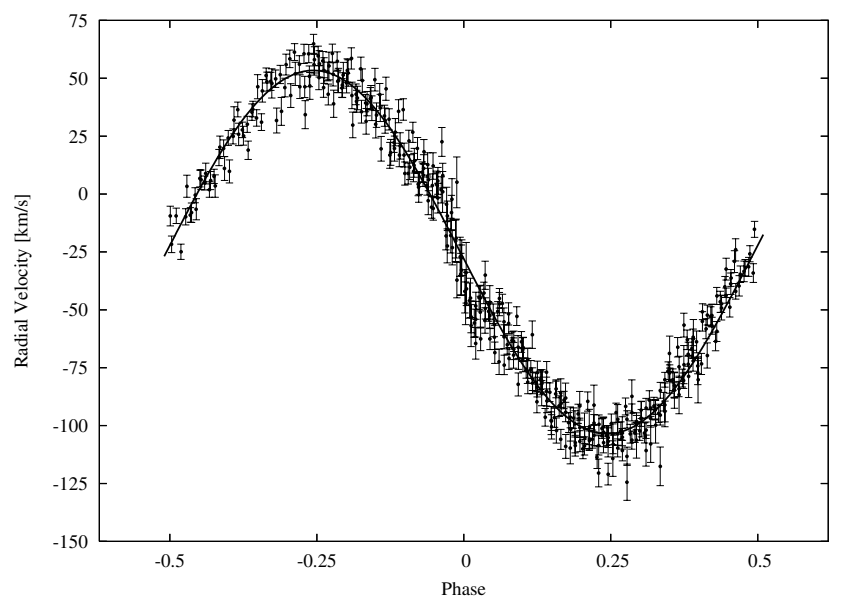

Fig. 4. The radial velocity measurements (average of the $\mathrm{H}_{\epsilon}, \mathrm{H}_{\delta}, \mathrm{H}_{\gamma}$ and $\mathrm{H}_{\beta}$ lines) of all the individual UVES/VLT spectra. The best fit orbit solution from PHOEBE is also shown.

A sample fit is shown in Fig. 3 for an individual spectrum. The FWHM of the two Gaussian fits, as well as their heights, were treated as a free parameter at first, but were kept fixed once the best fit values were found. We checked carefully if the RV from the $\mathrm{H}_{\gamma}$ line deviated from the one of the other Balmer lines, due to the discontinuity in its red wing. This turned out not to be the case (see also Fig. 3) so we kept the $\mathrm{H}_{\gamma} \mathrm{RV}$ values in our analysis.

Finally, the average of each RV measurement, using $\mathrm{H}_{\epsilon}, \mathrm{H}_{\delta}$, $\mathrm{H}_{\gamma}$ and $\mathrm{H}_{\beta}$ lines, was determined. These radial velocity values for each of the 399 individual spectra (with the errors), are shown in Fig. 4 together with the best fit orbital solution (see Sect. 4).

To perform an independent determination of the orbit from our spectroscopic data, and to verify the photometric ephemeris, the measured RVs (after barycentric correction of the velocities and the mid-exposure times) were subjected to a periodogram analysis. A sinusoidal fit using Period04 (Lenz \& Breger 2004) gives the frequency $114.25 \pm 0.1 \mu \mathrm{Hz}$ and the semi-amplitude $78.6 \pm 0.6 \mathrm{~km} \mathrm{~s}^{-1}$ which is, considering our poor frequency resolution of about $30 \mu \mathrm{Hz}$, in a good agreement with the orbital period $P=0.101015999$ d calculated by Kilkenny et al. (2000) as well as with the values derived from PHOEBE in Sect. 4 . The semi-amplitude of the velocity variation is in good agreement with the $78 \pm 3 \mathrm{~km} \mathrm{~s}^{-1}$ estimated by Kilkenny et al. (1998) (see their Table 4) even though they reported the semi-amplitudes of 
Table 1. Fixed parameters in the search for the orbital solution of PG 1336-018.

\begin{tabular}{ll}
\hline \hline Parameter & Value \\
\hline$t_{0}$ & $2450223.36134 \mathrm{~d}^{a}$ \\
$P$ & $0.101015999 \mathrm{~d}^{a}$ \\
$T_{\text {eff } 1}$ & $31300 \mathrm{~K}$ \\
$T_{\text {eff2 }}$ & $3000 \mathrm{~K}^{b}$ \\
$g_{1}$ & 1.0 \\
$g_{2}$ & 0.32 \\
$A_{1}$ & 1.0 \\
$x_{1}\left(g^{\prime}\right)$ & 0.217 \\
$x_{1}\left(r^{\prime}\right)$ & 0.178 \\
\hline
\end{tabular}

${ }^{a}$ Ephemeris taken from Kilkenny et al. (2000). ${ }^{b} T_{\text {eff2 }}$ was kept fixed as it is poorly constrained by the data, see the text for details.

all of their observations (see their Table 3 ) to range from $47 \pm 4$ to $79 \pm 4 \mathrm{~km} \mathrm{~s}^{-1}$. The semi-amplitude we obtained is somewhat larger than estimated by Woolf et al. (2003), $64 \pm 1 \mathrm{~km} \mathrm{~s}^{-1}$, but their data cover only 1.4 orbits and contain a gap which probably resulted in an underestimated value.

As our data set suffers from a baseline too short for reliable ephemeris determination, we adopted the ephemeris obtained by Kilkenny et al. (2000) (see Table 1).

Since the system is single-lined and the orbit is assumed to be circular, the analysis of the RV curve is straightforward. The mass function calculated from the semi-amplitude and the period gives:

$f(M)=0.0051 \pm 0.0001 M_{\odot}$.

\section{Orbital parameters}

In order to investigate the pulsational properties of PG 1336-018, the subject of a follow-up paper, the orbital variations due to the binarity must be removed from the observed lightcurve. However, in order to find the best orbital solution for this eclipsing binary system, the pulsations of the $\mathrm{sdB}$ primary must be removed as well. This is a non-trivial coupled problem. The determination of the orbital parameters of this system required to understand and evaluate the temporal spectrum of the primary $\mathrm{sdB}$ pulsator. In order to achieve this, we followed an iterative procedure, using all the information about the target we have. Once we find a reliable orbital solution, we subtract it from the lightcurves. Then we use the orbit subtracted lightcurves to extract the pulsation frequencies present in our data. We prewhiten the original observed lightcurves with these frequencies. The prewhitened lightcurves are then used as input to find the second iteration orbital solution.

\subsection{Fundamental parameters}

Our high resolution VLT/UVES spectra allow us to improve the spectroscopic parameters determined by Kilkenny et al. (1998). Using our RV solution (see Fig. 4), we shifted the spectra and added them together to improve the S/N. The coadded orbitsubtracted spectrum is shown in the bottom panel of Fig. 2.

For the model fitting procedure, we used the LTE models of Heber et al. (2000). The model spectra were convolved with a Gaussian instrumental profile of $0.25 \AA$ and rotationally broadened (assuming tidally locked rotation) with a $v \sin i$ of $74.2 \mathrm{~km} \mathrm{~s}^{-1}$. This produces a model spectrum with line cores that

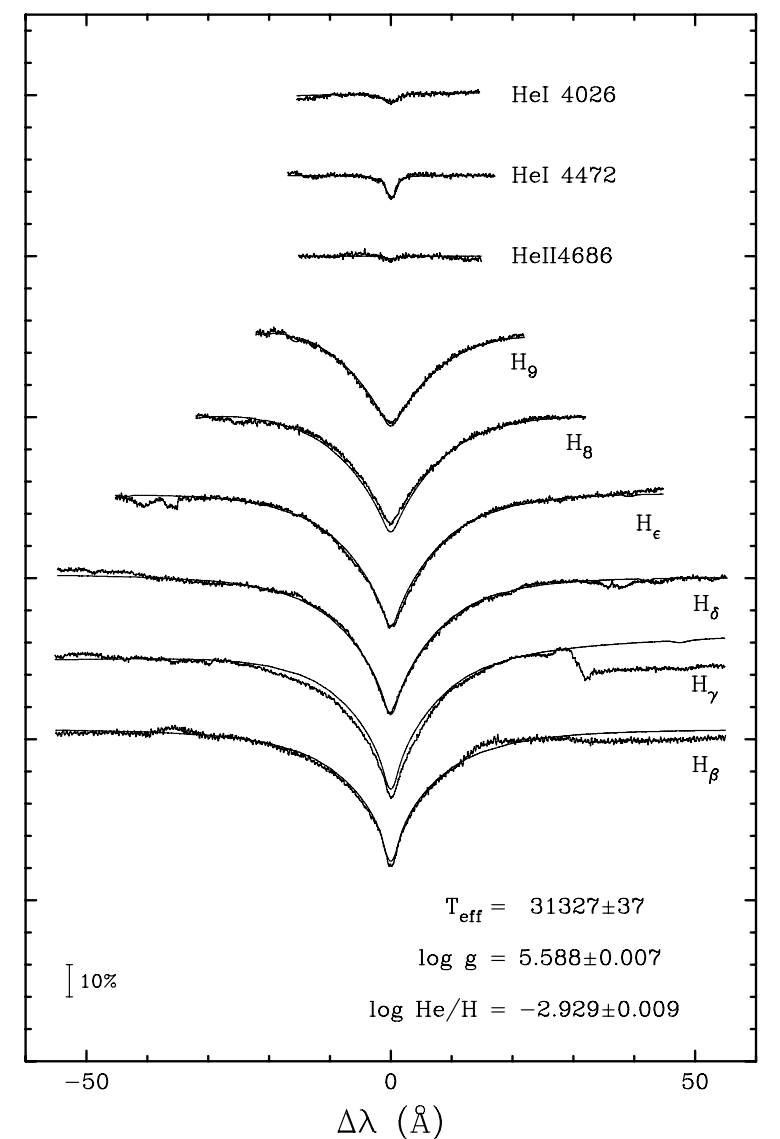

Fig. 5. Our spectroscopic model fit to the mean spectrum in Fig. 2. The best fit model spectrum has been plotted on top of the observed spectrum as a smooth curve. Note that the $\mathrm{H} \gamma$ line was kept out of the fit due to its proximity to an echelle order discontinuity.

reproduce the observed spectrum excellently for all lines that are unaffected by Echelle order discontinuities. Unfortunately, while the fit to the cores is good, the wings are not well fitted. Our best simultaneous fit for effective temperature, gravity and helium abundance yields:

$$
\begin{aligned}
T_{\mathrm{eff}} & =31300 \pm 250 \mathrm{~K} \\
\log g & =5.60 \pm 0.05 \mathrm{dex} \\
\log y & =-2.93 \pm 0.05 \mathrm{dex}
\end{aligned}
$$

The quoted errors are about five times larger than the formal fitting errors reported in Fig. 5. Although such $5 \sigma$ errors would normally be quite conservative considering the resolution and signal of the combined spectrum, there are obvious problems. The effects of errors due to the Echelle extraction problems described earlier are hard to quantify. The effective temperature is well constrained by the depth of the high order Balmer lines, and the helium abundance is determined by the depth of the narrow He I lines (marked in Figs. 2 and 5), which are not much affected by the Echelle extraction problems. However, since the Echelle order discontinuities strongly affect the wings of the lines, which are essential for the gravity determination, we cannot exclude a large error on $\log g$. For this reason, we will only use the effective temperature determination as a constraint for our orbital fitting procedure, and not $\log g$. Indeed, as we will see later, such a low $\log g$ is inconsistent with any realistic mass-radius relationship that can be derived from the orbit by at least 0.15 dex. In order to rule out other causes for the inconsistent $\log g$ determination from the average spectrum, we tried to fit it using NLTE 
atmosphere models, enhanced metallicity models, or changing the assumed rotational velocity broadening. All these attempts produced negligible changes to the derived parameters listed above.

\subsection{Binarity and pulsation}

Numerical orbit solutions were investigated using the PHOEBE package tool (Prša \& Zwitter 2005) which incorporates the aspects of the Wilson-Devinney (WD) code (Wilson \& Devinney 1971). The WD approach uses differential correction (DC) as the minimization method, which is in essence a linearised least squares method. The code was used in the mode for detached binaries with no constraints on the stellar potentials. No third light or spots were included.

The ULTRACAM/VLT $g^{\prime}$ and $r^{\prime}$ lightcurves and the RV measurements obtained from the UVES/VLT spectra were solved simultaneously to yield a consistent model fit. As PHOEBE is limited by the number of points (currently the limit is 9000 points) we had to phase bin our ULTRACAM/VLT lightcurves into 4000 data points per lightcurve.

The major problem in finding the orbital solution of any binary system is not only the fact that there are many free parameters $(12+5 n$, where $n$ is the number of lightcurves in different filters), but also that the parameters are correlated. Some of these correlations are severe, especially between the mass ratio $q$ and the potential of the secondary star $\Omega_{2}$ (see the discussion below in Sect. 4.3). Hence, one is left with several formal families of solutions within the parameter space. We must then confine the range of possible solutions by reducing the number of free parameters. The only safe way to do this is by considering the boundary conditions set by the data themselves and by sound theoretical considerations.

The parameters that were assumed and kept fixed in our analysis were $t_{0}, P, T_{\text {eff }}$ of the primary, gravity darkening coefficients both for the primary $g_{1}$ and the secondary $g_{2}$, bolometric albedo of the primary $A_{1}$ and the limb darkening coefficients of the primary in the two filters $x_{1}\left(g^{\prime}, r^{\prime}\right)$. For the gravity darkening coefficients we adopted values of 1.0 for the primary (radiative envelope) and 0.32 for the secondary (convective envelope). We assumed a circular orbit $(e=0)$ and synchronized rotation with the orbit.

The effective temperature of the primary $T_{\text {eff } 1}$ was set to the value derived from our spectra (see Sect. 4.1). The effective temperature previously estimated by Kilkenny et al. (1998, $T_{\text {eff }}=33000 \pm 1000$ ) was used as well, but, as it did not influence the derived parameters except for the luminosity of the stars, we fixed the temperature to the value derived by our new data. The $T_{\text {eff } 2}$ of the secondary has a very low contribution to the total flux (see Sect. 2) and, therefore, is not tightly constrained. An appropriate treatment of the effective temperature of the secondary in the case where the hot sdB primary is heating the cool secondary is not trivial, as the temperature on the illuminated hemisphere can be as much as five times higher than on the non-illuminated one (Zola 2000). Whilst we did not intend to fix the effective temperature of the secondary star at first, we have found that leaving it as an adjustable parameter does not give consistent results. With $T_{\text {eff } 2}$ as a free parameter, it converges to around $4000 \mathrm{~K}$ for the $g^{\prime}$ lightcurve, but to only $2700 \mathrm{~K}$ for the $r^{\prime}$-band lightcurve. As a reasonable compromise for $T_{\mathrm{eff} 2}$, we choose to fix it to $3000 \mathrm{~K}$. Considering the fact that the contribution of the secondary to the total flux is negligible, this is not an obstacle.

As there are no published limb darkening coefficients for $\mathrm{sdB}$ stars we calculated the limb darkening coefficients $x_{1}\left(g^{\prime}, r^{\prime}\right.$ and $u^{\prime}$ ) for a "typical" sdB star from a fully line-blanketed LTE model atmosphere (Behara \& Jeffery 2006) with $T_{\text {eff }}=$ $30000 \mathrm{~K}, \log g=5.5, V_{\text {turb }}=5 \mathrm{~km} \mathrm{~s}^{-1}$ and solar abundances (a linear cosine law was used). The mean limb darkening coefficients in each filter were computed by convolving the ULTRACAM efficiencies in each filter with the monochromatic limb darkening coefficients and the stellar fluxes. We also computed the orbital solution using an extrapolation of previously reported coefficients from the tables of Wade \& Rucinski (1985) and Al-Naimiy (1978), as well as the values fixed at 0.25 (V) and 0.20 (R) (Kilkenny et al. 1998). This did not change the solution, so we adopted the coefficients we computed from a modern atmosphere model. Table 1 summarises the values of the fixed parameters. The surface gravity is not a free parameter obtained by PHOEBE, since it is defined by the mass and radius.

Using the ephemeris given in Kilkenny et al. (2000) we find a phase shift of $0.00374 \pm 0.00006 \mathrm{~d}$. This phase shift could in principle be due to timing errors in our data rather than to an intrinsic change in the system. However, we carefully checked timings in our data sets and, moreover, we have data from two different instruments which both show the same phase shift. A timing error is therefore very unlikely to be the cause of the measured shift. A change inherent to the system is thus the most probable reason. With only two minima timings we cannot draw any further conclusion here, only emphasise the need for further epoch observations. A similar period change on the order of $0.003 \mathrm{~d}$ over a period of 6 years in the HW Vir system was documented by Kilkenny et al. (2000).

The strong pulsations in the lightcurves are obstructing the fine tuning of the orbit, as the pulsations are seen as scatter by PHOEBE. Therefore, we take the first iteration solution and subtract it from the lightcurves. Now, after the dominant parts of the periodicity, i.e. the eclipses, have been removed from the lightcurves we can analyse them in order to take out the pulsations of the primary from the lightcurves.

A Fourier amplitude spectrum was calculated for each orbit subtracted lightcurve to deduce the periodicities present in the data. The short timespan of our photometric data confines us with a frequency resolution of $54 \mu \mathrm{Hz}$. Since we are unable to resolve many of the closely spaced frequencies in the spectrum published by Kilkenny et al. (2003), we cannot use their peaks. We can only remove the periodicities we observe in our data in order to improve our orbit solution, after verifying that the frequencies we detect are indeed in the range of known PG 1336-018 frequencies.

After identifying the highest amplitude peak in the spectrum and cross-checking if this frequency is present in the previous data sets within our frequency resolution, we remove this peak from the data by subtracting a sine wave (with the frequency, amplitude and phase determined by a non-linear least-squares fit-NLLS) from the original lightcurves. We calculate the Fourier amplitude spectrum of the prewhitened residuals and repeat the procedure until no new peaks could be securely identified. In this way we are able to remove four frequencies, as listed in Table 2. The frequency spectrum of PG 1336-018 is complicated as there are many frequencies in a narrow frequency range, which are unresolved in our data set. Therefore the NLLS would not converge on a simultaneous fit to more than four frequencies, even though there is still significant power left in the Fourier spectrum. That is also the reason why the amplitudes appear higher in our data set compared to the ones seen in Kilkenny et al. (2003) as several frequencies are blended into one. The highest amplitude frequency in our data set at $5430.1 \mu \mathrm{Hz}$ is most probably the result of seven unresolved closely spaced 
Table 2. The list of frequencies, periods, amplitudes and phases we detected and prewhitened our data with. The phase is given as the time of maximum amplitude since $t_{0}$.

\begin{tabular}{|c|c|c|c|c|c|c|c|}
\hline $\begin{array}{c}\text { Frequency } \\
{[\mu \mathrm{Hz}]}\end{array}$ & $\begin{array}{c}\text { Period } \\
{[\mathrm{s}]}\end{array}$ & & $\begin{array}{c}\overline{\text { Amplitude }} \\
\text { [mma] }\end{array}$ & & & $\begin{array}{c}\text { hase }\left(T_{\max }\right. \\
{[\mathrm{s}]}\end{array}$ & \\
\hline & & $g^{\prime}$ & $r^{\prime}$ & $u^{\prime}$ & $g^{\prime}$ & $r^{\prime}$ & $u^{\prime}$ \\
\hline 5430.1 & 184.16 & $11.2(1)$ & $10.5(1)$ & $17.1(2)$ & $142.3(3)$ & $142.2(3)$ & 141.4(4) \\
\hline 5579.9 & 179.21 & $3.8(1)$ & $3.7(1)$ & $3.5(2)$ & $105.9(8)$ & 105.8(8) & $115(2)$ \\
\hline 5757.3 & 173.69 & $1.7(1)$ & $1.7(1)$ & $2.8(2)$ & $148(2)$ & $147(2)$ & $155(2)$ \\
\hline 7076.7 & 141.31 & $2.0(1)$ & $1.9(1)$ & $3.0(2)$ & $105(1)$ & $106(1)$ & $107(2)$ \\
\hline
\end{tabular}

Table 3. System parameters of the three best model fits to RV data and lightcurves of PG 1336-018. The formal $1 \sigma$ error on the last digit of each parameter is given in parentheses.

\begin{tabular}{|c|c|c|c|}
\hline Free parameter & Model I & Model II & Model III \\
\hline$a\left[R_{\odot}\right]$ & $0.723(5)$ & $0.764(5)$ & $0.795(5)$ \\
\hline & $0.282(2)$ & $0.262(2)$ & $0.250(2)$ \\
\hline$i\left[^{\circ}\right]$ & $80.67(8)$ & $80.67(8)$ & $80.67(8)$ \\
\hline$\Omega_{1}$ & $5.50(3)$ & $5.48(3)$ & $5.47(3)$ \\
\hline$\Omega_{2}$ & $2.77(1)$ & $2.68(1)$ & $2.62(1)$ \\
\hline$A_{2}$ & $0.92(3)$ & $0.92(3)$ & $0.93(3)$ \\
\hline$x_{2}\left(g^{\prime}\right)$ & $0.38(8)$ & $0.39(8)$ & $0.38(8)$ \\
\hline$x_{2}\left(r^{\prime}\right)$ & $0.88(8)$ & $0.89(8)$ & $0.89(8)$ \\
\hline \multicolumn{4}{|c|}{ Derived parameters: } \\
\hline$\overline{M_{1}}\left[M_{\odot}\right]$ & $0.389(5)$ & $0.466(6)$ & $0.530(7)$ \\
\hline$M_{2}\left[M_{\odot}\right]$ & $0.110(1)$ & $0.122(1)$ & $0.133(2)$ \\
\hline$R_{1}\left[R_{\odot}\right]$ & $0.14(1)$ & $0.15(1)$ & $0.15(1)$ \\
\hline$R_{2}\left[R_{\odot}\right]$ & $0.15(1)$ & $0.16(1)$ & $0.16(1)$ \\
\hline $\log g_{1}\left[\mathrm{~cm} / \mathrm{s}^{2}\right]$ & $5.74(5)$ & $5.77(6)$ & $5.79(7)$ \\
\hline $\log g_{2}\left[\mathrm{~cm} / \mathrm{s}^{2}\right]$ & $5.14(5)$ & $5.14(5)$ & $5.14(5)$ \\
\hline \multicolumn{4}{|c|}{ Roche radii: [in units of orbital separation] } \\
\hline$r_{1}($ pole $)$ & 0.191 & 0.191 & 0.191 \\
\hline$r_{1}$ (point) & 0.193 & 0.193 & 0.193 \\
\hline$r_{1}$ (side) & 0.192 & 0.192 & 0.192 \\
\hline$r_{1}$ (back) & 0.193 & 0.193 & 0.193 \\
\hline$r_{2}$ (pole) & 0.198 & 0.197 & 0.197 \\
\hline$r_{2}$ (point) & 0.213 & 0.215 & 0.216 \\
\hline$r_{2}($ side $)$ & 0.201 & 0.201 & 0.201 \\
\hline$r_{2}$ (back) & 0.210 & 0.211 & 0.211 \\
\hline \multicolumn{4}{|c|}{ Errors on residuals: } \\
\hline$\sigma\left(g^{\prime}\right)[\mathrm{mag}]$ & 0.03055 & 0.03054 & 0.03057 \\
\hline$\sigma\left(r^{\prime}\right)[\mathrm{mag}]$ & 0.01325 & 0.01321 & 0.01321 \\
\hline$\sigma(\mathrm{RV})\left[\mathrm{km} \mathrm{s}^{-1}\right]$ & 8.39 & 8.39 & 8.39 \\
\hline
\end{tabular}

frequencies $f_{3}, f_{4}, f_{25}, f_{10}, f_{5}, f_{7}$ and $f_{22}$ from Table 4 of Kilkenny et al. (2003).

These prewhitened lightcurves were then phase binned and, together with the RV curve, fed into PHOEBE to search for the improved orbit solution. Even though residual pulsations are still clearly visible in the lightcurves, their amplitudes are now significantly smaller, which allows us to obtain a more reliable (second iteration) orbit solution. A third iteration step turns out to be unnecessary, as it does not improve the final outcome of the orbital parameters.

As a quantitative measure of the goodness-of-fit we use the $1 \sigma$ deviation for each data set $\left(g^{\prime}, r^{\prime}\right.$ and RV) from the simultaneously calculated synthetic curves. The bigger $1 \sigma$ deviation in $g^{\prime}$ is due to the higher amplitudes of the oscillations in this colour. While it is impossible to see the depth of the local minima found by the DC method, and therefore search for the global minimum of the parameter hyperspace, we tested the stability of the convergent solutions found by parameter kicking (Prša $\&$ Zwitter 2005). Once convergence was reached, we manually kicked the parameters and the minimization was restarted from the displaced points. In this way we found three groups of solutions of equal goodness-of-fit. Table 3 gives the three best fit orbital solutions. It is not possible to decide which solution is the correct one based on the numerical considerations as the synthetic curves are fitting the data equally well for all three models. The errors given in the table are the formal errors of the fit which are likely smaller than the true errors due to the above mentioned correlation between the parameters. The synthetic lightcurve fits to the observed data points are presented in Figs. 4, 6 and 7 (solid line) together with their residuals. The synthetic $g^{\prime}$ and $r^{\prime}$ lightcurves and the RV curve are plotted for only one solution (Model II) since the deviations between the three solutions cannot be resolved at the scale of the figure.

\subsection{Discussion}

The uniqueness of a given solution is jeopardized by the parameter correlations. In particular, there is a strong correlation between the mass ratio $q$ and the potential of the secondary star $\Omega_{2}$. Therefore, there is a $q$ degeneracy in all the orbital solutions. For a given range of potentials defined by the Lagrangian point, a family of solutions with corresponding mass ratios is found. The solutions found in Table 3 represent the local minima shown in Fig. 8.

The relative radii and the orbital inclination are tightly constrained by the depth and the width of the eclipses, and the results in all three models are nearly identical. There is only a slight distortion of the secondary: $r_{2}$ (pole) $/ r_{2}$ (point) is 0.93 , $0.92,0.91$ respectively for each model. While the previous searches for the best orbital solutions (Kilkenny et al. 1998; Drechsel et al. 2001, and references therein) tend to resort to non-physical albedos (greater that 1 in some cases) and limb darkening coefficients of the secondary, we find that the biggest problem is in the temperature of the secondary which is heated by the hot subdwarf. The weakest point of all modelling procedures lies in an inadequate treatment of the temperature of the secondary star. The temperature distribution over the surface of the secondary has to be incorporated in the atmosphere models used by PHOEBE in order to get more realistic solutions. This is far beyond the scope of our current paper.

The surface gravity derived from the orbital solutions, although in agreement with the value previously estimated by Kilkenny et al. $(1998, \log g=5.7 \pm 0.1 \mathrm{dex})$ is higher than the spectroscopic gravity estimate. Therefore, we have explored the full range of mass-radius ranges for the primary allowed by the orbital solution and the spectroscopic gravity (Fig. 9). The parameters used to generate this orbital solution mass-radius relationship are only the $P, i, K_{1}$ and the radius of the primary in terms $a$, none of which are affected by the $q$ degeneracy. Thus, if we had a sufficiently accurate spectroscopic determination of $\log g$, we could use the relationships in Fig. 9 to determine one unique $M_{1}$. Unfortunately, our spectroscopic $\log g$ of 5.6 is 


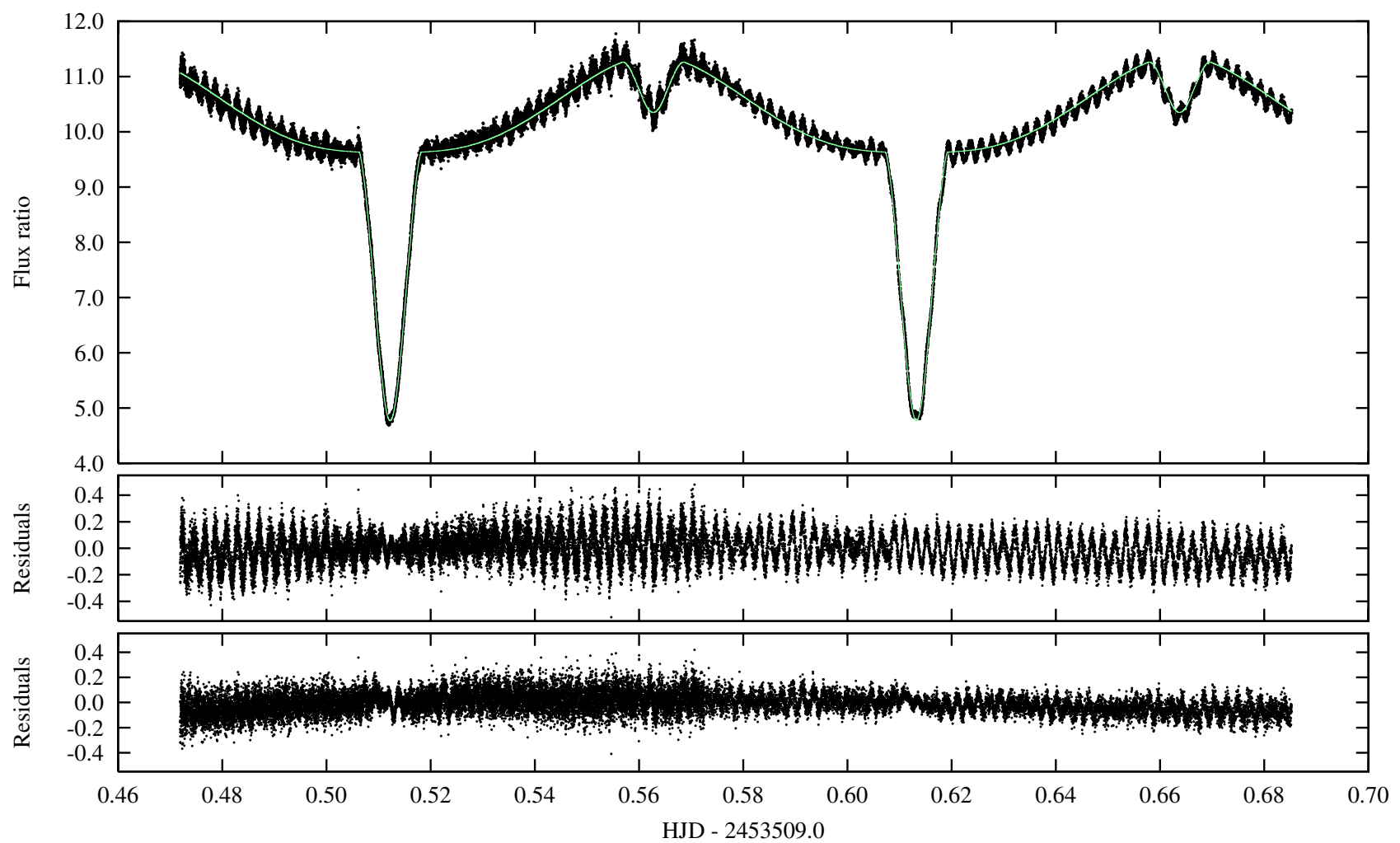

Fig. 6. The ULTRACAM/VLT $g^{\prime}$ lightcurve together with the synthetic orbit solution. The middle panel shows the residuals of the orbit subtraction. Pulsations during the eclipses are now clearly visible, and we can see that the amplitude is smaller during the primary eclipse than during the secondary as only the part of the surface is visible. The bottom panel shows the residuals after prewhitening with the four strongest oscillation modes.

clearly much lower than what can realistically be accepted since it gives a mass for the primary that is far too low $\left(M_{1}<0.2 M_{\odot}\right)$.

While we cannot discriminate between the three model fits on the basis of their $\sigma$ values, the evolutionary scenarios for $\mathrm{sdB}$ stars disqualify the Model III solution as the primary mass would be too high for a core He-flash (Han et al. 2002). Models I and II however, are both possible as they could have formed through common envelope phase (Hu et al., submitted to A\&A).

\section{Detection of the Rossiter-McLaughlin effect}

In Fig. 4, an apparent up-and-down (redshift-blueshift) shift occurs at phase zero in the RV curve. This effect at the eclipse is known as the Rossiter-McLaughlin (RM) effect (Rossiter 1924; McLaughlin 1924). It is due to the selective blocking of the light of the rotating star during an eclipse. When the secondary star covers the blueshifted (redshifted) half of the stellar disk, the integrated light of the primary appears slightly redshifted (blueshifted). Because of this selective blocking of the stellar surface during the eclipse, a skewed line profile is created. This change in line profile shape results in a shift in RV, which in turn results in the redshift-blueshift distortion seen during the eclipse (see Fig. 4). The RM effect has been seen in other eclipsing hot subdwarf binaries (e.g. AA Dor: Rauch \& Werner 2003) and can be used to investigate the rotational properties of the component stars. It was recently used in extrasolar planetary transits (Queloz et al. 2000; Ohta et al. 2005; Giménez 2006; Gaudi \& Winn 2007) to discriminate between different migration theories. The amplitude of the effect mainly depends on the projected rotation velocity of the star, the ratio of stellar radii, the orbital inclination, and the limb darkening.
To analyze this effect we have subtracted the orbital solution (solid curve in Fig. 4) from the RV measurements. The orbitsubtracted RV residuals, phase binned in 50 bins, are plotted in Fig. 10. The RM effect is clearly seen in these residuals. We used the analytical description of this effect given in Giménez (2006) to simulate the RM effect for this system. We have assumed that the rotational axis of the primary star is co-aligned with the perpendicular to the orbital plane. The result of this simulation is plotted as a solid line in Fig. 10. The equatorial rotational velocity of the star was set to $75.2 \mathrm{~km} \mathrm{~s}^{-1}$ and the ratio of the stellar radii $r_{2} / r_{1}$, the inclination of the orbit $i$ and the radius of the primary relative to the size of the orbit $r_{1}$ were taken from our orbital solution (see Table 3). The synthetic curve fits the observed RM amplitude rather well. The uncertainties on the residual RV curve are too large to fine-tune the orbital parameters. We can only establish that the observed RM effect is compatible with the orbital solutions given in Table 3 and represents an independent confirmation of the light curve solution.

The apparent asymmetry seen in Fig. 10 is, however, not well explained. Such an asymmetry is expected to occur if the projected orbital and rotational axes are not aligned. This is highly unlikely for the narrow orbit of PG 1336-018. Nevertheless, we simulated the RM effect allowing different angles of the rotation axes and the orbital axes. We indeed could not achieve satisfactory results, because, when the zero offset was fitted well, the amplitudes were highly asymmetrical and vice versa. The asymmetry is more likely caused by the pulsations seen during the primary eclipse, which also give rise in line profile shape variations. The equations describing the RM effect assume that the components are spherical, i.e. they do not take into account any deviation from spherical symmetry such as the one produced by 


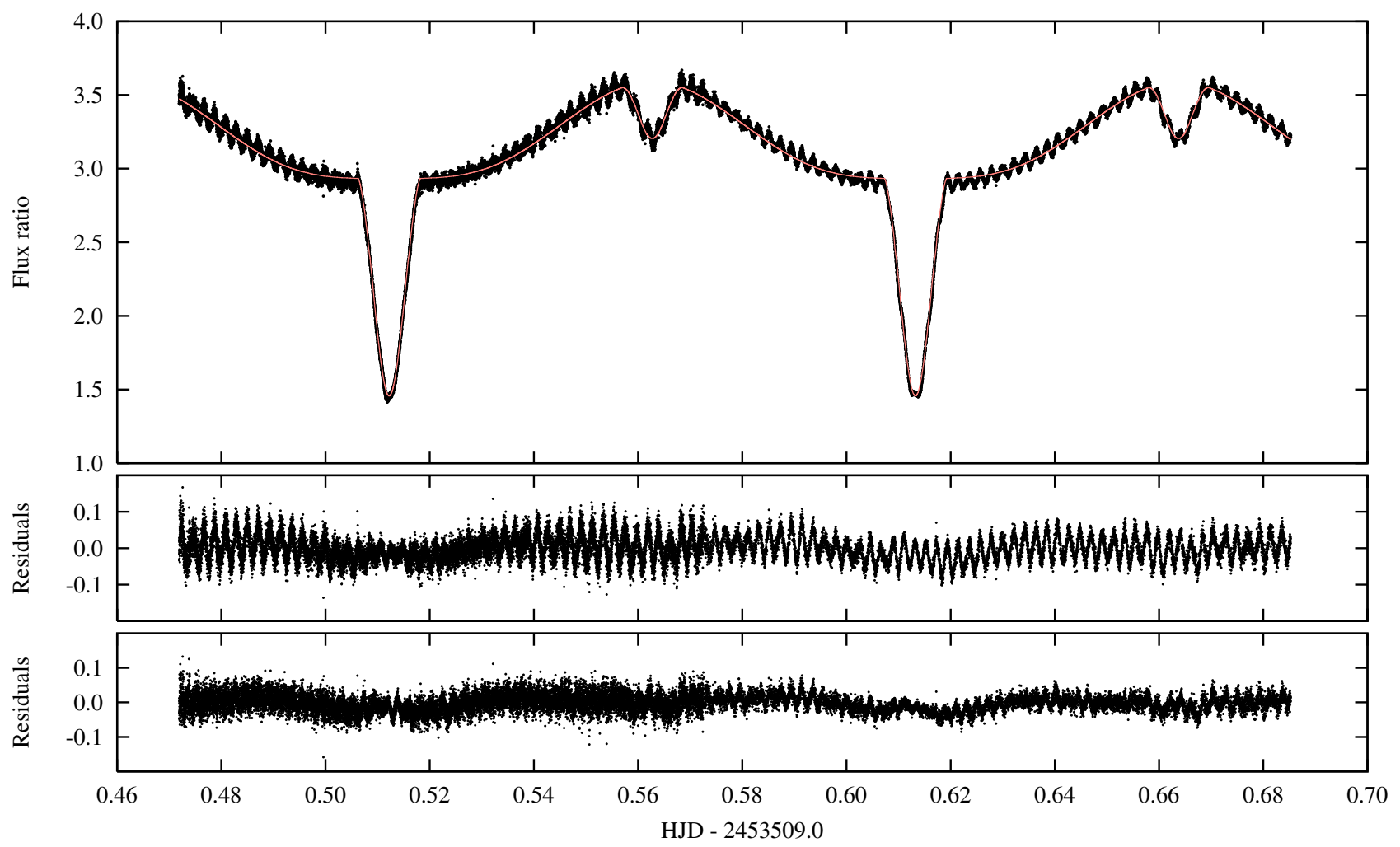

Fig. 7. Same as Fig. 6 but for the $r^{\prime}$ band. The trends seen in the middle and bottom panel result from imperfect removal of the reflection effect due to the changing temperature across the surface of the secondary (see text for details).

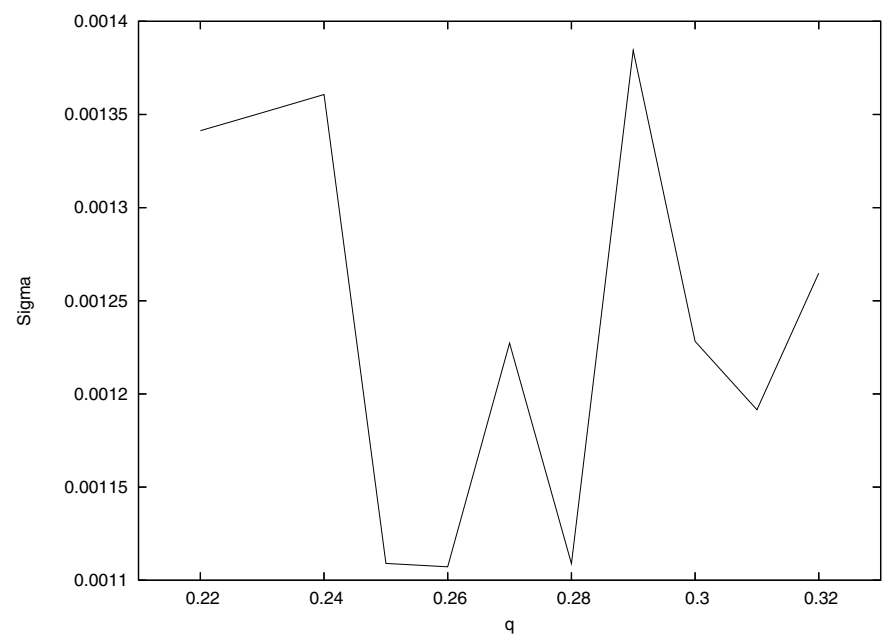

Fig. 8. Mass ratio $q$ versus sigma, for the range of the possible $q$ values. Sigma is the sum of the squares of the sigmas in the two considered filters $\left(\sigma\left(g^{\prime}\right)\right.$ and $\left.\sigma\left(r^{\prime}\right)\right)$.

the pulsations. We will investigate this further in our follow-up paper dedicated to the analysis of the primary's pulsations.

\section{Conclusions and future work}

In this work, we presented a thorough observational analysis of the orbital behavior of the pulsating eclipsing binary PG 1336-018. Our goal was to avoid using a canonical mass of $0.5 M_{\odot}$ for the subdwarf in any interpretation of the luminosity variations of the star, as has been done so far in the literature.Instead, we attempted an unbiased derivation of the system and stellar parameters, in particular for the masses of the

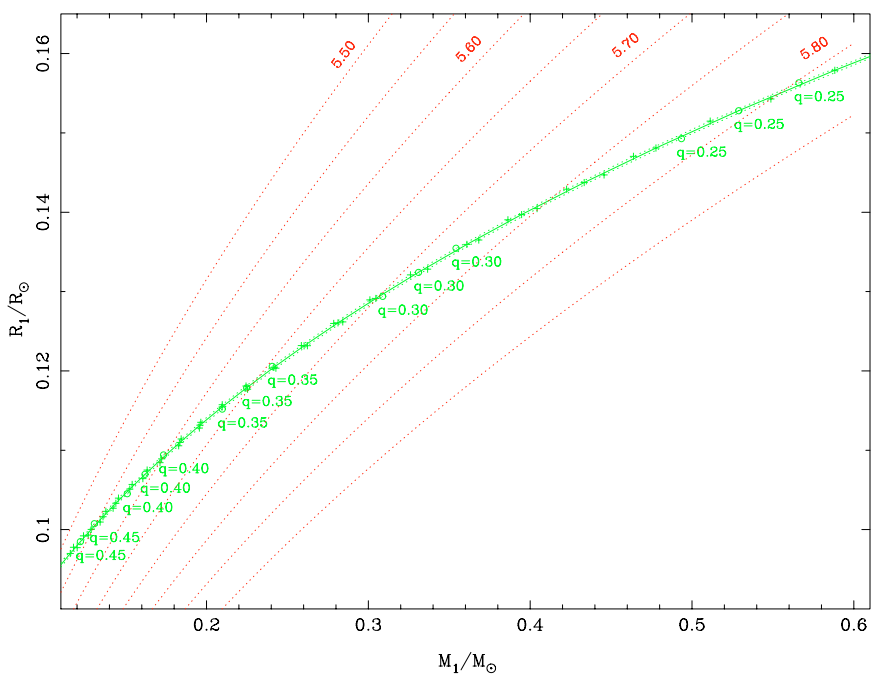

Fig. 9. Mass-radius diagram for PG 1336-018 showing the regions permitted by the orbit solution (continuous line) and by the different surface gravities (dotted lines). The $q$ values are also noted on the orbit solution. The small changes from the $3 \sigma$ error on $K_{1}$ do not shift the curve representing the orbital solution.

components. Our analysis resulted in three equally probable sets of orbital and physical parameters of the system. Our model III solution is incompatible with the binary having gone through a core He-flash and a common-envelope phase described by the $\alpha$-formalism since that can only lead to PG $1336-018$ like binaries with primary masses up to $0.48 M_{\odot}$ (Hu et al., submitted to A\&A). This leaves us with two solutions, one with a primary mass of $0.466 \pm 0.006 M_{\odot}$ and another with $0.389 \pm 0.005 M_{\odot}$, with secondary masses of $0.122 \pm 0.001 M_{\odot}$ and $0.110 \pm 0.001 M_{\odot}$ 


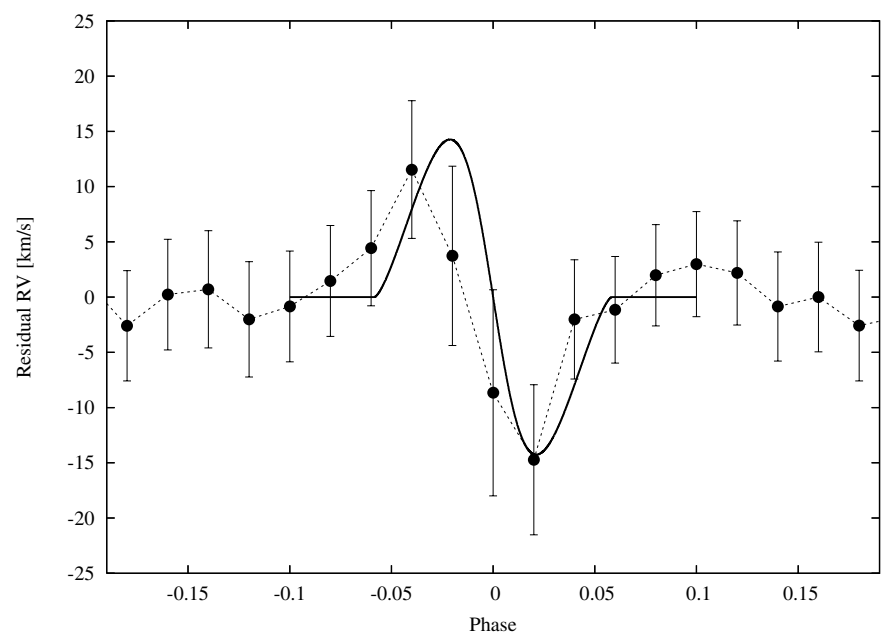

Fig. 10. The orbit subtracted RV residuals (dots) with their corresponding errors clearly showing the RM effect. The solid line is the simulation of the RM effect with the parameters given in the text.

respectively. We thus conclude that our solutions with $M_{1}=$ $0.466 \pm 0.006 M_{\odot}$ and $M_{1}=0.389 \pm 0.005 M_{\odot}$ are the only plausible ones, except when the common-envelope phase would be better described by the $\gamma$-formalism (Nelemans et al. 2000; Nelemans \& Tout 2005). In this case all three solutions are acceptable, as this formalism allows non-degenerate helium ignition with a broader primary mass range $\left(0.3-0.8 M_{\odot}\right)$.

Furthermore, we have detected the RM effect in the radial velocity curve of PG 1336-018. The simulated amplitude of the $\mathrm{RM}$ effect is in the accordance with the RM amplitude seen in the RV residuals, which is an independent confirmation of the results obtained from our orbital solution.

While deriving the orbital solution for PG 1336-018, we hit upon the limitation of current binary analysis codes, which also prevented us to pinpoint the effective temperature of the secondary. None of the analysis methods available in the literature treat the atmosphere of such a close binary, in which one component is so hot that it induces a temperature gradient across the surface of the other, in an appropriate way. Indeed, all codes make use of stellar atmosphere models which assume one fixed effective temperature at the surface of each of the component stars. As such, any derived quantities, such as limb darkening coefficients and albedos, cannot be but a very crude approximation of reality whenever one component is seriously heated by the other one. In the case of close binaries like PG 1336-018, i.e. with a hot primary and a cold secondary, the temperature of the latter changes so drastically from the illuminated side to the backside, that specific atmosphere models representing such a situation should be computed and used while deriving the orbital parameters. This is an entire project by itself and surely beyond the scope of our current work. We hope that our results will give rise to future developments of atmosphere models with temperatures varying across the surface of the cool component in close binaries. The case of PG 1336-018, and our data of the star, are ideally suited to test such new future models.

In a follow-up paper of this work, we plan to analyse the oscillatory signal in our multicolour photometry and highresolution spectroscopy, after the orbit subtraction presented here. This will be done by computing a cross-correlation function of each spectrum and investigating the signature of the modes in it. Cross-correlation functions have already been used to study the character of oscillations modes before, see e.g.
Mathias \& Aerts (1996) for the $\delta$ Scuti star 20 CVn and Hekker et al. (2006) for solar-like oscillations in red giants. This is done by computing line diagnostics, such as moments, and the amplitude and phase across the profile, and comparing these to predictions based on the theory of non-radial oscillations. In principle, this allows us to identify the spherical wavenumbers $(\ell, m)$ of the strongest modes. The use of these established mode identification techniques (see e.g. Briquet \& Aerts 2003; Zima 2006, for the latest versions) on high-resolution cross-correlation profiles of pulsating sdB stars has so far not yet been done. The nature of our data and of our target star requires a simulation study to test the effects of smearing out the oscillations over the cycle and of the limited time base. Also, we must treat the data during and outside the eclipses separately in order to assess the effectiveness of the techniques in the specific case of PG 1336-018. Such a study is currently being performed. The ultimate goal of it is to identify the highest-amplitude modes and discriminate among the plausible seismic models of the star. This will then eventually lead us to derive a seismic mass estimate to be confronted with the observed primary masses presented here and with the evolutionary masses computed by $\mathrm{Hu}$ et al. (submitted to A\&A).

Acknowledgements. M.V. thanks Maarten Reyniers for his generous help in the UVES data reduction procedure. M.V. acknowledges a Ph.D. scholarship from the Research Council of Leuven University. H.H. acknowledges a Ph.D. scholarship through the "Convenant Katholieke Universiteit Leuven, Belgium - Radboud Universiteit Nijmegen, the Netherlands". M.V., C.A., R.Ø., and H.H. are supported by the Research Council of Leuven University, through grant GOA/2003/04. ULTRACAM is supported by PPARC grants $\mathrm{PPA} / \mathrm{G} / \mathrm{S} / 2003 / 00058$ and PP/D002370/1. We are thankfull to Joshua Winn and Scott Gaudi for enlightening discussions on the RM effect and to Alvaro Giménez for kindly providing us with his subroutines. We thank Prof. Uli Heber for kindly providing the LTE spectral grids.

\section{References}

Aerts, C., \& Eyer, L. 2000, in Delta Scuti and Related Stars, ed. M. Breger, \& M. Montgomery, ASP Conf. Ser., 210, 113

Al-Naimiy, H. M. 1978, Ap\&SS, 53, 181

Behara, N. T., \& Jeffery, C. S. 2006, A\&A, 451, 643

Brassard, P., Fontaine, G., Billères, M., et al. 2001, ApJ, 563, 1013

Briquet, M., \& Aerts, C. 2003, A\&A, 398, 687

Charpinet, S., Fontaine, G., Brassard, P., \& Dorman, B. 1996, ApJ, 471, L103

Charpinet, S., Fontaine, G., Brassard, P., \& Dorman, B. 2000, ApJS, 131, 223

Charpinet, S., Fontaine, G., Brassard, P., Green, E. M., \& Chayer, P. 2005, A\&A, 437,575

Dhillon, V., \& Marsh, T. 2001, New Astronomy Review, 45, 91

Dorman, B., Rood, R. T., \& O'Connell, R. W. 1993, ApJ, 419, 596

Drechsel, H., Heber, U., Napiwotzki, R., et al. 2001, A\&A, 379, 893

Dreizler, S., Koester, D., \& Heber, U. 2000, Baltic Astronomy, 9, 113

Dupret, M.-A., De Ridder, J., De Cat, P., et al. 2003, A\&A, 398, 677

Fukugita, M., Ichikawa, T., Gunn, J. E., et al. 1996, AJ, 111, 1748

Fusi-Pecci, F., \& Renzini, A. 1976, A\&A, 46, 447

Gaudi, B. S., \& Winn, J. N. 2007, ApJ, 655, 550

Giménez, A. 2006, ApJ, 650, 408

Green, E. M., Fontaine, G., Reed, M. D., et al. 2003, ApJ, 583, L31

Green, R. F., Schmidt, M., \& Liebert, J. 1986, ApJS, 61, 305

Han, Z., Podsiadlowski, P., Maxted, P. F. L., Marsh, T. R., \& Ivanova, N. 2002, MNRAS, 336, 449

Han, Z., Podsiadlowski, P., Maxted, P. F. L., \& Marsh, T. R. 2003, MNRAS, 341, 669

Heber, U., Reid, I. N., \& Werner, K. 2000, A\&A, 363, 198

Hekker, S., Aerts, C., de Ridder, J., \& Carrier, F. 2006, A\&A, 458, 931

Jeffery, C. S., Aerts, C., Dhillon, V. S., Marsh, T. R., \& Gänsicke, B. T. 2005, MNRAS, 362, 66

Kilkenny, D., Koen, C., O’Donoghue, D., \& Stobie, R. S. 1997, MNRAS, 285, 640

Kilkenny, D., O’Donoghue, D., Koen, C., Lynas-Gray, A. E., \& van Wyk, F. 1998, MNRAS, 296, 329

Kilkenny, D., Keuris, S., Marang, F., et al. 2000, The Observatory, 120, 48 Kilkenny, D., Reed, M. D., O’Donoghue, D., et al. 2003, MNRAS, 345, 834 Lenz, P., \& Breger, M. 2004, in IAU Symposium, ed. J. E. A. Zverko, 786 Mathias, P., \& Aerts, C. 1996, A\&A, 312, 905 
Maxted, P. f. L., Heber, U., Marsh, T. R., \& North, R. C. 2001, MNRAS, 326, 1391

McLaughlin, D. B. 1924, ApJ, 60, 22

Menzies, J. W., \& Marang, F. 1986, in Instrumentation and Research Programmes for Small Telescopes, ed. J. B. Hearnshaw, \& P. L. Cottrell, IAU Symp., 118, 305

Morales-Rueda, L., Maxted, P. F. L., Marsh, T. R., North, R. C., \& Heber, U. 2003, MNRAS, 338, 752

Morales-Rueda, L., Maxted, P. F. L., Marsh, T. R., Kilkenny, D., \& O’Donoghue, D. 2006, Baltic Astron., 15, 187

Mukai, K. 1990, PASP, 102, 183

Napiwotzki, R., Karl, C. A., Lisker, T., et al. 2004, Ap\&SS, 291, 321

Nelemans, G., \& Tout, C. A. 2005, MNRAS, 356, 753

Nelemans, G., Verbunt, F., Yungelson, L. R., \& Portegies Zwart, S. F. 2000, A\&A, 360, 1011

Ohta, Y., Taruya, A., \& Suto, Y. 2005, ApJ, 622, 1118
Østensen, R., Oreiro, R., Drechsel, H., Heber, U., \& Pigulski, A. 2007, in ASP Conf. Ser., 15th European Workshop on White Dwarfs, ed. R. Napiwotzki, in press

Prša, A., \& Zwitter, T. 2005, ApJ, 628, 426

Queloz, D., Eggenberger, A., Mayor, M., et al. 2000, A\&A, 359, L13

Randall, S. K., Fontaine, G., Brassard, P., \& Bergeron, P. 2005, ApJS, 161, 456

Rauch, T., \& Werner, K. 2003, A\&A, 400, 271

Rossiter, R. A. 1924, ApJ, 60, 15

Wade, R. A., \& Rucinski, S. M. 1985, A\&AS, 60, 471

Wilson, R. E., \& Devinney, E. J. 1971, ApJ, 166, 605

Wood, J. H., Zhang, E.-H., \& Robinson, E. L. 1993, MNRAS, 261, 103

Woolf, V. M., Jeffery, C. S., \& Pollacco, D. 2003, in NATO ASIB Proc. 105, White Dwarfs, ed. D. E. A. de Martino, 95

Zima, W. 2006, A\&A, 455, 227

Zola, S. 2000, Baltic Astron., 9, 197 